\title{
Polarization phase gate with a tripod atomic system
}

\author{
S. Rebić, ${ }^{1, *}$ D. Vitali, ${ }^{1}$ C. Ottaviani, ${ }^{1}$ P. Tombesi,${ }^{1}$ M. Artoni ${ }^{2,3}$ F. Cataliotti, ${ }^{3,4}$ and R. Corbalán ${ }^{5}$ \\ ${ }^{1}$ INFM and Dipartimento di Fisica, Università di Camerino, I-62032 Camerino, Italy \\ ${ }^{2}$ INFM and Dipartimento di Chimica e Fisica dei Materiali, Università di Brescia, I-25133 Brescia, Italy \\ ${ }^{3}$ European Laboratory for Non-linear Spectroscopy, via N. Carrara, I-50019 Sesto Fiorentino, Italy \\ ${ }^{4}$ INFM and Dipartimento di Fisica, Università di Catania, I-95124 Catania, Italy \\ ${ }^{5}$ Departament de Física, Universitat Autònoma de Barcelona, E-08193, Bellaterra, Spain
}

(Received 20 February 2004; published 21 September 2004)

\begin{abstract}
We analyze the nonlinear optical response of a four-level atomic system driven into a tripod configuration. The large cross-Kerr nonlinearities that occur in such a system are shown to produce nonlinear phase shifts of order $\pi$. Such a substantial shift may be observed in a cold atomic gas in a magneto-optical trap where it could be feasibly exploited towards the realization of a polarization quantum phase gate. The experimental feasibility of such a gate is here examined in detail.
\end{abstract}

DOI: 10.1103/PhysRevA.70.032317

PACS number(s): 03.67.Pp, 42.65.-k, 42.50.Gy

\section{INTRODUCTION}

A great effort has recently gone into the search for practical architecture for quantum information processing systems. While most attention has been devoted toward theoretical issues, several strategies have also been proposed for experimental investigations. However, the laboratory demand for building quantum information devices are quite severe, requiring strong coupling between qubits, the quantum carriers of information, in an environment with minimal dissipation. For this reason experimental progress has so far lagged behind the remarkable development that quantum information theory now witnesses [1].

Here we focus on optical implementations of quantum information processing systems. Traveling optical pulses are the natural candidates for the realization of quantum communication schemes and many experimental demonstrations of quantum key distribution [2,3] and quantum teleportation schemes [4-8] have been already performed. Optical systems have been also proposed for the implementation of quantum computing, even though the absence of significant photon-photon interactions is an obstacle for the realization of efficient two-qubit quantum gates, which are needed for implementing universal quantum computation [1]. Various schemes have been proposed to circumvent this problem. One is linear optics quantum computation [9], which is a probabilistic scheme based on passive linear optical devices, efficient single photon sources, and detectors, and which implicitly exploits the nonlinearity hidden in the photodetection process (see Refs. $[10,11]$ for some preliminary demonstrations of this scheme). Other schemes explicitly exploit optical nonlinearities for quantum gate implementations. Typical optical nonlinearities are too small to provide a substantial photon-photon interaction, hence limiting the usefulness of an all-optical quantum gate. However, there seems to be a way to overcome the problem. Quantum interference effects associated with electromagnetically induced transparency

*Email address: stojan.rebic@unicam.it
(EIT) [12-14] have quite recently been shown to enhance these nonlinearities by as much as 10 orders of magnitude [15]. This enhancement is commonly exhibited by a weak probe beam in the presence of another strong coupling beam when both impinge off-resonance on a three-level atomic sample at very low temperatures.

The off-resonance condition is rather crucial to the observation of the enhancement and one can, in general, identify two ways for attaining that. One is to introduce an additional laser beam whose detuning from a fourth level is larger than the level linewidth [16]. In this " $N$ " configuration one of the ground levels undergoes an ac-Stark shift which disturbs the EIT resonance condition and induces an effective Kerr nonlinearity while keeping absorption negligible. Improvements by many orders of magnitude with respect to conventional nonlinearities have indeed been observed in this way [17]. In addition, strong cross-phase modulation [16] and photon blockade (i.e., strong self-phase modulation) have also been predicted [18-22].

Another and related way to obtain large nonlinearities consists in disturbing the exact two-photon resonance condition in a $\Lambda$ configuration. This can be achieved by slightly mismatching the probe and coupling field frequencies yet remaining within the EIT transparency window making the dispersion of the probe field not exactly zero. In this case enhanced Kerr nonlinearities have been observed in the $\Lambda$ configuration $[23,24]$ and predicted in the so-called chain- $\Lambda$ configurations [25-29]. By using this second approach Ottaviani et al. [29] have shown that large cross-phasemodulations that occur in an " $\mathrm{M}$ " configuration may lead to an all-optical two-qubit quantum phase gate (QPG) [1,30], where one qubit gets a phase shift dependent on the state of the other qubit. Here, the key element enabling large crossphase modulation is the possibility of group velocity matching. Large cross-phase modulations occur when two optical pulses, a probe and a trigger, interact for a sufficiently long time. This happens when their group velocities are both small and comparable [31,32] and there exists several ways by which this can be done [29,31,33].

In this paper we propose an alternative phase gating scheme that can greatly reduce, when compared with other 
schemes, the experimental effort for its realizability. As also done in Ref. [29], the binary information is encoded in the polarization degree of freedom of a probe and a trigger optical pulse while the phase-gate mechanism relies on an enhanced cross-phase modulation effect which occurs in a relatively simple and robust four atomic level tripod configuration. The scheme requires good control over frequencies and intensities of the probe and trigger laser pulses.

Optical QPG have been already experimentally studied. A conditional phase shift $\phi \simeq 16^{\circ}$ between two frequencydistinct high- $Q$ cavity modes, due to the effective cross modulation mediated by a beam of Cs atoms, has been measured in Ref. [34]. However, the complete truth table of the gate has not been determined in this experiment. A conditional phase shift $\phi \simeq 8^{\circ}$ has been instead obtained between weak coherent pulses, using a second-order nonlinear crystal [35]. However, this experiment did not demonstrate a bona fide QPG because $\phi$ depends on the input states, and the gate can be defined only for a restricted class of inputs.

The four-level tripod configuration that we adopt here has been extensively studied in the past few years. For example, Unanyan et al. [36] used a tripod configuration to achieve stimulated Raman adiabatic passage (STIRAP) for creating an arbitrary coherent superposition of two atomic states in a controlled way. Paspalakis et al. [37-39], in particular, developed the interesting possibility of using a tripod scheme for efficient nonlinear frequency generation. Moreover, it was shown that the group velocity of a probe pulse may be significantly reduced, as in the familiar $\Lambda$ system [37]. The work of Malakyan [40] was the first to suggest that the tripod scheme may be used to entangle a pair of very weak optical fields in an atomic sample. This work has been recently extended to the case of quantum probe and trigger fields in Ref. [41], where an adiabatic treatment similar to that of Ref. [31] is adopted.

The purpose of this paper is thus twofold. First, we adopt a standard density-matrix approach, including spontaneous emission and dephasings, to analyse the nonlinear optical response of a four-level tripod configuration. In particular, we examine the conditions under which large cross-Kerr nonlinearities may occur in a cold atomic sample. Second, we study the possibility of employing such an enhanced cross-phase-modulation to devise a polarization phase-gating mechanism which turns out to be rather robust and apt to actual experimental investigations.

The paper is organized as follows. In Sec. II, dressed states of the atomic tripod are analyzed and their significance emphasized. In Sec. III, we solve the set of Bloch equations and derive expressions for linear and nonlinear susceptibilities. In Sec. IV group velocity matching is discussed in detail, while Sec. V discusses the operation of a polarization phase gate. We summarize our results in Sec. VI.

\section{DRESSED STATES OF THE TRIPOD SYSTEM}

The tripod configuration as shown in Fig. 1 enables one to achieve a giant cross-phase-modulation between probe and trigger fields. Here transitions $|1\rangle \rightarrow|0\rangle$ and $|3\rangle \rightarrow|0\rangle$ are driven by a probe and trigger fields of respective Rabi fre- $|0\rangle$

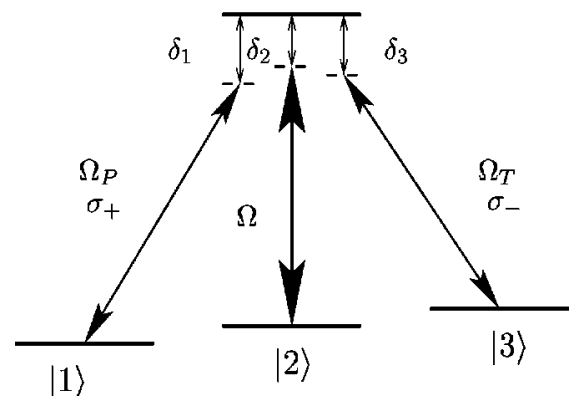

FIG. 1. Energy level scheme for a tripod. Probe and trigger fields have Rabi frequencies $\Omega_{P}$ and $\Omega_{T}$ and polarizations $\sigma_{+}$and $\sigma_{-}$. The pump Rabi frequency is $\Omega$ while $\delta_{j}=\omega_{0}-\omega_{j}-\omega_{j}^{(L)}$ denote the laser (frequency $\omega_{j}^{(L)}$ ) detunings from the respective transitions $|j\rangle \leftrightarrow|0\rangle$.

quencies $\Omega_{P}$ and $\Omega_{T}$, while the transition $|2\rangle \rightarrow|0\rangle$ is driven by a control field of Rabi frequency $\Omega$. Moreover, $\delta_{j}=\omega_{0}$ $-\omega_{j}-\omega_{j}^{(L)}$ denote the laser (frequency $\omega_{j}^{(L)}$ ) detunings from the respective transitions $|j\rangle \leftrightarrow|0\rangle$. In the interaction picture and in the dipole and rotating wave approximations the tripod Hamiltonian is given by

$$
\begin{aligned}
\mathcal{H}_{\text {int }}= & \hbar \delta_{1} \sigma_{00}+\hbar\left(\delta_{1}-\delta_{2}\right) \sigma_{22}+\hbar\left(\delta_{1}-\delta_{3}\right) \sigma_{33}+\hbar\left(\Omega_{P}^{*} \sigma_{10}\right. \\
& \left.+\Omega_{P} \sigma_{01}\right)+\hbar\left(\Omega^{*} \sigma_{20}+\Omega \sigma_{02}\right)+\hbar\left(\Omega_{T}^{*} \sigma_{30}+\Omega_{T} \sigma_{03}\right),
\end{aligned}
$$

where $\sigma_{i j}=|i\rangle\langle j|$ are pseudospin atomic operators. Spontaneous emission and dephasing are not relevant at this stage but will be added [42] later in Eqs. (4) below.

Minimal conditions required for the generation of such a large nonlinear effect can be assessed through a straightforward analysis in terms of dressed states which we here briefly outline. When the three detunings are equal, i.e., $\delta_{i}$ $=\delta(i=1,2,3)$, two of the four eigenstates of Hamiltonian (1) [36], namely

$$
\left|e_{ \pm}\right\rangle=\frac{\Omega_{P}|1\rangle \pm|0\rangle+\Omega_{T}|3\rangle+\Omega|2\rangle}{\sqrt{\Omega_{P}^{2}+\Omega^{2}+\Omega_{T}^{2}}},
$$

have energies $\delta \pm \sqrt{\delta^{2}+\Omega_{P}^{2}+\Omega^{2}+\Omega_{T}^{2}}$, while the other two with equal energy $\delta$ are degenerate and acquire the form (written here for a special case $\delta=0$ ),

$$
\begin{gathered}
\left|e_{1}\right\rangle=\frac{\Omega_{T}|1\rangle-\Omega_{P}|3\rangle}{\sqrt{\Omega_{P}^{2}+\Omega_{T}^{2}}}, \\
\left|e_{2}\right\rangle=\frac{\Omega \Omega_{P}|1\rangle+\Omega \Omega_{T}|3\rangle-\left(\Omega_{P}^{2}+\Omega_{T}^{2}\right)|2\rangle}{\sqrt{\left(\Omega_{P}^{2}+\Omega_{T}^{2}\right)\left(\Omega_{P}^{2}+\Omega^{2}+\Omega_{T}^{2}\right)}} .
\end{gathered}
$$

These two are dark states as neither of them contains contributions from the excited state $|0\rangle$. Notice, in addition, that for different detunings the two dark states are no longer degenerate as their energies become, respectively, $\delta_{2}$ and $\delta_{3}$.

Necessary conditions for achieving a large cross-Kerr phase shift can be formulated as follows: (i) probe and trigger must be tuned to dark states, (ii) the transparency fre- 
quency window for each of these dark states has to be narrow and with a steep dispersion to enable significant group velocity reduction, and finally (iii) there must be a degree of symmetry between the two transparency windows so that trigger and probe group velocities can be made to be equal $[29,31,33]$. These conditions can be satisfied by taking all three detunings nearly equal. By slightly departing from the exact resonance condition, in fact, and for frequency mismatches within the transparency window, strong cross-Kerr modulations along with group velocity matching can be achieved. This, as we will show below, will enable us to realize an efficient phase-gate operation. It is also worth noticing that for perfectly equal detunings degeneracy leads to a common transparency window for both fields; in this case the nonlinear susceptibility contributions in Eqs. (9) vanish and our tripod system becomes linear.

\section{BLOCH EQUATIONS AND SUSCEPTIBILITIES}

The Bloch equations for the density matrix elements (including atomic spontaneous emission and dephasing) are

$$
\begin{gathered}
i \dot{\rho}_{00}=-i\left(\gamma_{11}+\gamma_{22}+\gamma_{33}\right) \rho_{00}+\Omega_{P}^{*} \rho_{10}-\Omega_{P} \rho_{01}+\Omega^{*} \rho_{20} \\
-\Omega \rho_{02}+\Omega_{T}^{*} \rho_{30}-\Omega_{T} \rho_{03}, \\
i \dot{\rho}_{11}=i \gamma_{11} \rho_{00}+i \gamma_{12} \rho_{22}+i \gamma_{13} \rho_{33}+\Omega_{P} \rho_{01}-\Omega_{P}^{*} \rho_{10} \\
i \dot{\rho}_{22}=i \gamma_{22} \rho_{00}-i \gamma_{12} \rho_{22}+i \gamma_{23} \rho_{33}+\Omega \rho_{02}-\Omega^{*} \rho_{20}, \\
i \dot{\rho}_{33}=i \gamma_{33} \rho_{00}-i\left(\gamma_{13}+\gamma_{23}\right) \rho_{33}+\Omega_{T} \rho_{03}-\Omega_{T}^{*} \rho_{30}, \\
i \dot{\rho}_{10}=-\Delta_{10} \rho_{10}+\Omega_{P} \rho_{00}-\Omega_{P} \rho_{11}-\Omega_{12}-\Omega_{T} \rho_{13}, \\
i \dot{\rho}_{20}=-\Delta_{20} \rho_{20}+\Omega \rho_{00}-\Omega \rho_{22}-\Omega_{P} \rho_{21}-\Omega_{T} \rho_{23}, \\
i \dot{\rho}_{30}=-\Delta_{30} \rho_{30}+\Omega_{T} \rho_{00}-\Omega_{P} \rho_{33}-\Omega_{P} \rho_{31}-\Omega \rho_{32}, \\
i \dot{\rho}_{12}=-\Delta_{12} \rho_{12}+\Omega_{P} \rho_{02}-\Omega^{*} \rho_{10}, \\
i \dot{\rho}_{13}=-\Delta_{13} \rho_{13}+\Omega_{P} \rho_{03}-\Omega_{T}^{*} \rho_{10}, \\
i \dot{\rho}_{23}=-\Delta_{23} \rho_{23}+\Omega \rho_{03}-\Omega_{T}^{*} \rho_{20},
\end{gathered}
$$

where $\rho_{i j}=\operatorname{Tr}\left\{\sigma_{j i} \rho\right\}=\langle i|\rho| j\rangle$. Decay rates $\gamma_{i j}$ describe decay of populations and coherences, $\Delta_{j 0}=\delta_{j}+i \gamma_{j 0}$ and $\Delta_{i j}=\delta_{j}-\delta_{i}$ $-i \gamma_{i j}$, with $i, j=1,2,3$.

We consider the steady-state solutions to the Bloch equations. When the intensity of the pump field is stronger than the intensity of both probe and trigger $|\Omega|^{2} \gg\left|\Omega_{P, T}\right|^{2}$, and the detunings and decay rates are of the same order of magnitude, the stationary population distribution will be symmetric with respect to the $1 \leftrightarrow 3$ exchange, i.e., $\rho_{11} \approx \rho_{33} \approx 1 / 2$, with the population of the other two levels vanishing. Note that this is an assumption on steady-state populations only, not the choice of particular dressed state. It is also consistent with the numerical solution of the full set of Bloch equations (4).

This population assumption allows to decouple the equations for the populations from those of the coherences and to obtain the steady-state solution for the latter, yielding the probe and trigger susceptibilities according to

$$
\begin{aligned}
& \chi_{P}=-\lim _{t \rightarrow \infty} \frac{\mathcal{N}\left|\boldsymbol{\mu}_{P}\right|^{2}}{\hbar \epsilon_{0}} \frac{\rho_{10}(t)}{\Omega_{P}}, \\
& \chi_{T}=-\lim _{t \rightarrow \infty} \frac{\mathcal{N}\left|\boldsymbol{\mu}_{T}\right|^{2}}{\hbar \epsilon_{0}} \frac{\rho_{30}(t)}{\Omega_{T}},
\end{aligned}
$$

where $\mathcal{N}$ is the atomic density and $\boldsymbol{\mu}_{P, T}$ the electric dipole matrix elements for probe and trigger transitions, respectively. Rabi frequencies are defined in terms of electric-field amplitudes $E_{P, T}$ as $\Omega_{P, T}=-\left(\boldsymbol{\mu}_{P, T} \cdot \boldsymbol{\varepsilon}_{P, T}\right) E_{P, T} / \hbar$, with $\boldsymbol{\varepsilon}_{P, T}$ being the polarization unit vector of probe and trigger beams. The resulting general expression for the steady-state $(s s)$ probe and trigger susceptibilities are obtained from

$$
\begin{aligned}
\frac{\left(\rho_{10}\right)_{s s}}{\Omega_{P}}= & \left(1+\frac{1}{4} \frac{\left(\Delta_{12} \Delta_{23} / \Delta_{13}^{2}\right)\left|\Omega_{P}\right|^{2}\left|\Omega_{T}\right|^{2}}{\left(\Delta_{10} \Delta_{12}-|\Omega|^{2}\right)\left(\Delta_{30}^{*} \Delta_{23}-|\Omega|^{2}\right)}\right)^{-1} \\
& \times\left\{-\frac{1}{2} \frac{\Delta_{12} \Delta_{13}}{\Delta_{10} \Delta_{12} \Delta_{13}-\left.\Delta_{13}\left|\Omega^{2}-\Delta_{12}\right| \Omega_{T}\right|^{2}}\right. \\
& \left.-\frac{1}{2} \frac{\Delta_{12} \Delta_{13} \Delta_{23}\left|\Omega_{T}\right|^{2}}{\Delta_{30}^{*} \Delta_{13} \Delta_{23}-\Delta_{13}|\Omega|^{2}-\Delta_{23}\left|\Omega_{P}\right|^{2}}\right\}, \\
\frac{\left(\rho_{30}\right)_{s s}}{\Omega_{T}}= & \left(1+\frac{1}{4} \frac{\left(\Delta_{23}^{*} \Delta_{12}^{*} / \Delta_{13}^{* 2}\left|\Omega_{P}\right|^{2}\left|\Omega_{T}\right|^{2}\right.}{\left(\Delta_{30} \Delta_{23}^{*}-|\Omega|^{2}\right)\left(\Delta_{10}^{*} \Delta_{12}^{*}-|\Omega|^{2}\right)}\right)^{-1} \\
& \times\left\{-\frac{1}{2} \frac{\Delta_{23}^{*} \Delta_{13}^{*}}{\Delta_{30} \Delta_{23}^{*} \Delta_{13}^{*}-\Delta_{13}^{*}|\Omega|^{2}-\Delta_{23}^{*}\left|\Omega_{P}\right|^{2}}\right. \\
& \left.-\frac{1}{2} \frac{\Delta_{23}^{*} \Delta_{13}^{*} \Delta_{12}^{*}\left|\Omega_{P}\right|^{2}}{\Delta_{10}^{*} \Delta_{13}^{*} \Delta_{12}^{*}-\Delta_{13}^{*}|\Omega|^{2}-\Delta_{12}^{*}\left|\Omega_{T}\right|^{2}}\right\} .
\end{aligned}
$$

We are interested in the cross-phase-modulation between the probe and trigger fields. Therefore, we keep the two lowest order contributions in trigger and probe: linear and thirdorder nonlinear susceptibilities, while neglecting the higher orders in the expansion. This yields

$$
\begin{aligned}
& \chi_{P}=\chi_{P}^{(1)}+\chi_{P}^{(3)}\left|E_{T}\right|^{2}, \\
& \chi_{T}=\chi_{T}^{(1)}+\chi_{T}^{(3)}\left|E_{P}\right|^{2},
\end{aligned}
$$

that is, each susceptibility has a linear and a cross-Kerr nonlinear term, while self-phase modulation terms are of higher order. Both susceptibilities have a linear contribution because of the nonzero stationary population in levels $|1\rangle$ and $|3\rangle$. Linear susceptibilities are given by

$$
\chi_{P}^{(1)}=\frac{\mathcal{N}\left|\boldsymbol{\mu}_{P}\right|^{2}}{\hbar \epsilon_{0}} \frac{1}{2} \frac{\Delta_{12}}{\Delta_{10} \Delta_{12}-|\Omega|^{2}},
$$




$$
\chi_{T}^{(1)}=\frac{\mathcal{N}\left|\boldsymbol{\mu}_{T}\right|^{2}}{\hbar \epsilon_{0}} \frac{1}{2} \frac{\Delta_{23}^{*}}{\Delta_{30} \Delta_{23}^{*}-|\Omega|^{2}},
$$

where the factor $1 / 2$ in each equation comes from the symmetric steady-state population distribution. The cross-Kerr susceptibilities are instead given by

$$
\begin{aligned}
\chi_{P}^{(3)}= & \mathcal{N} \frac{\left|\boldsymbol{\mu}_{P}\right|^{2}\left|\boldsymbol{\mu}_{T}\right|^{2}}{\hbar^{3} \epsilon_{0}} \times \frac{1}{2} \frac{\Delta_{12} / \Delta_{13}}{\Delta_{10} \Delta_{12}-|\Omega|^{2}} \\
& \times\left(\frac{\Delta_{12}}{\Delta_{10} \Delta_{12}-|\Omega|^{2}}+\frac{\Delta_{23}}{\Delta_{30}^{*} \Delta_{23}-|\Omega|^{2}}\right), \\
\chi_{T}^{(3)}= & \mathcal{N} \frac{\left|\boldsymbol{\mu}_{T}\right|^{2}\left|\boldsymbol{\mu}_{P}\right|^{2}}{\hbar^{3} \epsilon_{0}} \times \frac{1}{2} \frac{\Delta_{23}^{*} / \Delta_{13}^{*}}{\Delta_{30} \Delta_{23}^{*}-|\Omega|^{2}} \\
& \times\left(\frac{\Delta_{12}^{*}}{\Delta_{10}^{*} \Delta_{12}^{*}-|\Omega|^{2}}+\frac{\Delta_{23}^{*}}{\Delta_{30} \Delta_{23}^{*}-|\Omega|^{2}}\right) .
\end{aligned}
$$

Note that Eqs. (8) and also Eqs. (9) are completely symmetric with respect to the $1 \leftrightarrow 3$ exchange. ${ }^{1}$ This exchange symmetry is ensured by the complex conjugate terms in Eqs. (8b) and $(9 \mathrm{~b})$ and it is expected because of the symmetry of the population distribution. Note also that in the absence of dephasing, the nonlinear susceptibility has a singularity at $\delta_{1}=\delta_{3}$. The necessary regularization is provided by a nonzero dephasing term $i \gamma_{13}$.

Paspalakis and Knight [37] have recently analyzed the properties of the tripod system in a somewhat different setup. It is nevertheless instructive to compare the results of this section with theirs. In the scheme of Ref. [37], population is assumed to be initially in the ground state $|1\rangle$. Provided that $\left|\Omega_{P}\right|^{2} \ll|\Omega|^{2},\left|\Omega_{T}\right|^{2}$ population remains in $|1\rangle$ in the steady state. Paspalakis and Knight calculate the expression for probe susceptibility to the first order in $\Omega_{P}$. It is easy to see that their expression is consistent (up to a factor $1 / 2$ determined by the different population distribution) to our result in Eq. (6a): considering only terms to the first order in $\Omega_{P}$ leaves only the first term in the curly brackets of Eq. (6b). Additional terms in Eqs. (6) arise because we are looking for a cross-Kerr nonlinearity in both probe and trigger, so that all the terms of third order have to be included. We also note that it is the manipulation of eigenstate $\left|e_{1}\right\rangle$ [Eq. (3a)] that is responsible for parametric processes of Refs. [37-39].

\section{GROUP VELOCITY MATCHING}

The linear and nonlinear susceptibilities of Eqs. (8) and (9) have all the properties required for a large cross-phase modulation. In fact, our tripod system can be seen as formed by two adjacent $\Lambda$ systems, one involving the probe field and one involving the trigger field, sharing the same control field. Therefore both fields exhibit EIT, which here manifests itself through the presence of two generally distinct transparency windows, corresponding to the two dark states of Eq. (3). Perfect EIT for both fields takes place when the two trans-

\footnotetext{
${ }^{1}$ The full symmetry also requires $\left|\boldsymbol{\mu}_{T}\right|^{2}=\left|\boldsymbol{\mu}_{P}\right|^{2}$, which is fulfilled for the proposed ${ }^{87} \mathrm{Rb}$ scheme, see Sec. V.
}
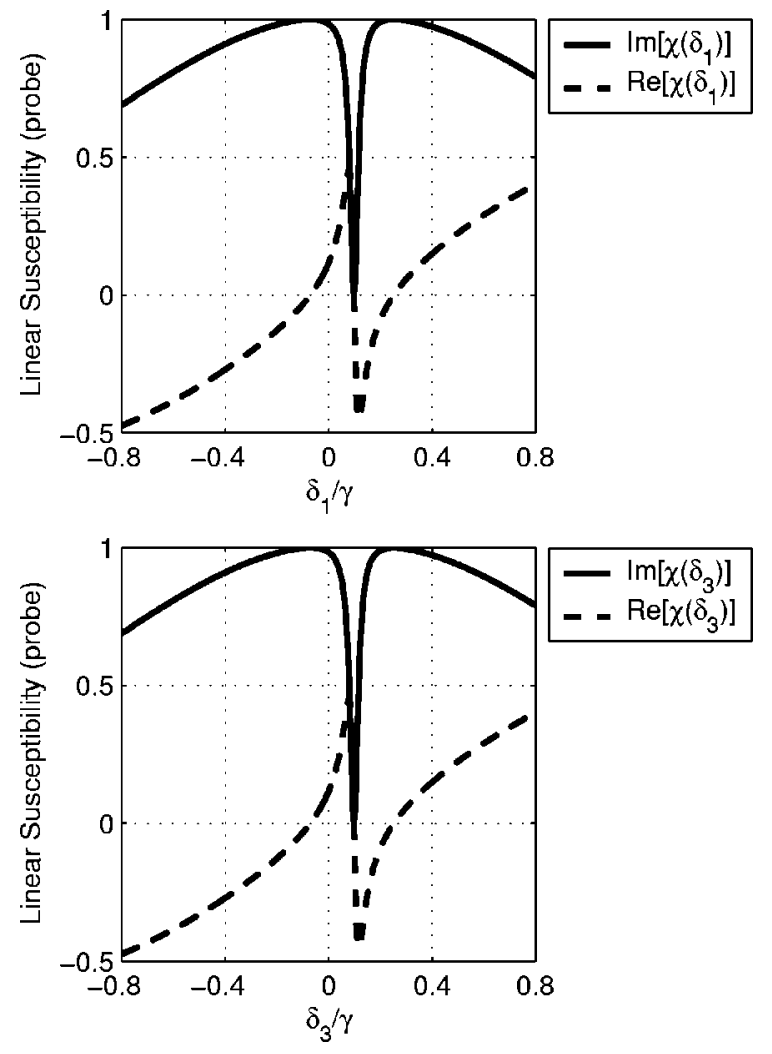

FIG. 2. Probe absorption and dispersion $\chi\left(\delta_{1}\right)$ $=\hbar \epsilon_{0} \chi_{P} /\left(\mathcal{N}\left|\boldsymbol{\mu}_{P}\right|^{2}\right)=\left(\rho_{10} / \Omega_{P}\right)_{s s}\left[\gamma^{-1}\right]$ (upper frame) vs the probe detuning $\delta_{1} / \gamma$ when $\delta_{3}=0.1 \gamma$ and $\delta_{2}=0.1 \gamma$. Trigger absorption and dispersion $\chi\left(\delta_{3}\right)=\hbar \epsilon_{0} \chi_{T} /\left(\mathcal{N}\left|\boldsymbol{\mu}_{T}\right|^{2}\right)=\left(\rho_{30} / \Omega_{T}\right)_{s s}\left[\gamma^{-1}\right]$ (lower frame) vs the trigger detuning $\delta_{3} / \gamma$ when $\delta_{1}=0.1 \gamma$ and $\delta_{2}=0.1 \gamma$. In both cases we take the Rabi frequencies as $\Omega_{P}=\Omega_{T}=0.1 \gamma, \Omega=\gamma$.

parency windows coincide, i.e., when the two dark states are degenerate, which is achieved when the three detunings $\delta_{i}$ are all equal. In this case, all physical effects related to standard EIT are present and in particular the steep dispersion responsible for the reduction of the group velocity which is at the basis of the giant cross-Kerr nonlinearity (see Fig. 2). The condition of equal detunings (exact double EITresonance condition) is important also for another reason. In fact, together with the symmetry of Eqs. (8) and (9) with respect to the $1 \leftrightarrow 3$ exchange, it also guarantees identical dispersive properties for probe and trigger and therefore the same group velocity. As first underlined by Lukin and Imamoğlu [31], group velocity matching is another fundamental condition for achieving a large nonlinear mutual phase shift because only in this way the two optical pulses interact in a transparent nonlinear medium for a sufficiently long time.

The group velocity of a light pulse is given in general by $v_{g}=c /\left(1+n_{g}\right)$, where $c$ is the speed of light in vacuum and

$$
n_{g}=\frac{1}{2} \operatorname{Re}[\chi]+\frac{\omega_{0}}{2}\left(\frac{\partial \operatorname{Re}[\chi]}{\partial \omega}\right)_{\omega_{0}}
$$

is the group index, $\omega_{0}$ being the laser frequency. The group index of Eq. (10) is essentially determined by the linear susceptibility $\chi^{(1)}$, because contributions from the nonlinear 
terms are orders of magnitude smaller and can be neglected. Using Eqs. (8), it is possible to get a simple expression for the two group velocities in the case of equal detunings. This condition corresponds to the center of the transparency window for each field, where $\operatorname{Re}\left[\chi^{(1)}\right]$ vanishes, and the group velocity is reduced due to a large dispersion gradient. One has

$$
\begin{aligned}
& \left(v_{g}\right)_{P} \approx \frac{4 \hbar c \epsilon_{0}}{\omega_{P} \mathcal{N}\left|\boldsymbol{\mu}_{P}\right|^{2}}\left(|\Omega|^{2}+\left|\Omega_{T}\right|^{2}\right), \\
& \left(v_{g}\right)_{T} \approx \frac{4 \hbar c \epsilon_{0}}{\omega_{T} \mathcal{N}\left|\boldsymbol{\mu}_{T}\right|^{2}}\left(\left|\Omega_{P}\right|^{2}+|\Omega|^{2}\right),
\end{aligned}
$$

so that, as expected from the $1 \leftrightarrow 3$ symmetry, group velocity matching is achieved for $\left|\Omega_{P}\right|=\left|\Omega_{T}\right|$.

Unfortunately, it is possible to check from Eqs. (9) that when $\delta_{i}=\delta, \forall i$ exactly, the system becomes linear, i.e., the real part of the nonlinear susceptibilities vanish and there is no cross-phase modulation. This means that we have to "disturb" the exact EIT resonance conditions, by taking slightly different detunings. This is a general conclusion, valid for any atomic level scheme resembling multiple $\Lambda$ systems [26-29]. If the double EIT-resonance condition is disturbed by a small amount, one remains within the common transparency window and the absorption is still negligible. Moreover, the two group velocities can be matched also in the nonresonant case. In fact, from the symmetry of Eqs. (8), one has that the gradients-and hence the group velocities-can be kept symmetric and all the conclusions for the exact resonance remain valid in the vicinity of resonance as well.

\section{PHASE GATE OPERATION}

A significant cross-phase-modulation is the key ingredient for the implementation of a quantum phase gate between two optical qubits. Such a cross-phase-modulation could be realized exploiting the cross-Kerr effect whereby an optical field acquires a phase shift conditioned to the state of another optical field. The relevant gate transformation is defined through the following input-output relations $|i\rangle_{1}|j\rangle_{2}$ $\rightarrow \exp \left\{i \phi_{i j}\right\}|i\rangle_{1}|j\rangle_{2}$, where $i, j=0,1$ denote the qubit basis. In particular, this becomes a universal two-qubit gate, that is a gate able to entangle two initially factorized qubits, when the conditional phase shift $\phi=\phi_{11}+\phi_{00}-\phi_{10}-\phi_{01}$ becomes different from zero $[1,30,34]$.

A natural choice for encoding binary information in optical beams consists in using the polarization degree of freedom, in which case the two logical basis states $|0\rangle$ and $|1\rangle$ of the above gate transformation correspond to two orthogonal light polarizations. A possible experimental implementation can be realized with the tripod scheme discussed above by using ${ }^{87} \mathrm{Rb}$ atoms confined in a temporal dark SPOT (spontaneous-force optical trap). This is a magneto-optical trap (MOT) where the repumping beam has been temporarily shut off [43]. In such a trap cold atoms are transferred in the $\left|5 S_{1 / 2}, F=1, m=\{-1,0,1\}\right\rangle$ state(s) of ${ }^{87} \mathrm{Rb}$ while density is increased with respect to a conventional MOT. In this case states $|1\rangle,|2\rangle$, and $|3\rangle$ correspond to the ground-state Zeeman sublevels $\left|5 S_{1 / 2}, F=1, m=\{-1,0,1\}\right\rangle$, and state $|0\rangle$ corresponds to the excited state $\left|5 P_{3 / 2}, F=0\right\rangle$. The atoms are available for just a few milliseconds which is long compared with the typical microsecond time scales involved in our proposed experiment.

A universal QPG could be implemented when a significant and nontrivial cross-phase-modulation between probe and trigger fields arises but only for one of the four input probe and trigger polarization configurations. This occurs for our tripod configuration of Fig. 1 only when the probe is $\sigma^{+}$ polarized and the trigger is $\sigma^{-}$polarized. When the probe has instead a $\sigma^{-}$polarization [Eq. (18a)], that is to say the "wrong" polarization, there is no sufficiently close level it may couple to and hence the corresponding pulse will acquire the trivial (vacuum) phase shift $\phi_{0}^{P}=k_{P} l$, where $l$ is the length of the medium. The trigger pulse with the "correct" $\sigma^{-}$ polarization, on the other hand, will acquire in this case the linear phase shift

$$
\phi_{\text {lin }}^{T}=k_{T} l\left(1+2 \pi \chi_{T}^{(1)}\right) .
$$

It is worth noticing here that for sufficiently narrow probe and trigger laser linewidths and nearly equal detunings as used in our scheme, cross-phase modulation between the two $\sigma^{-}$polarized probe and trigger pulses [Eq. (18a)] does not occur for sublevels Zeeman shifts larger than (half) the EIT transparency bandwidth. Owing to the fact that such a bandwidth is typically smaller than $\gamma(\sim 0.1 \gamma$ in our case $)$, crossKerr nonlinearities for the case of a wrong probe polarization [Eq. (18a)] can readily be avoided for sufficiently large Zeeman splittings. ${ }^{2}$ Such a splitting should further be chosen so as to make a $\sigma^{-}$polarized probe transparent [44], namely, by making it falling outside the absorption profile of both trigger transition (see Figs. 2 and 3) and any other nearby resonance. For the specific rubidium levels configuration that we examine below, Zeeman shifts of about $20-30 \gamma$ should be appropriate to avoid simultaneous absorption and cross-Kerr nonlinearities of a wrongly polarized probe. ${ }^{3}$ The correct shift to be used would clearly depend on the value of the probe and trigger detunings. ${ }^{4}$ The case of a wrong $\sigma^{+}$polarized trigger [Eq. (18c)] can be discussed in just the same way leading to a vacuum shift $\phi_{0}^{T}$ and to a linear shift $\phi_{\text {lin }}^{P}$ which obtain from the $\phi_{0}^{P}$ and $\phi_{\text {lin }}^{T}$ above upon interchanging $P \leftrightarrow T$. When probe and trigger both have the "wrong" polarization, i.e., the probe is $\sigma^{-}$polarized and the trigger is $\sigma^{+}$polarized, there is no sufficiently close level to which probe and trigger

\footnotetext{
${ }^{2}$ Right and wrong polarizations are distinguished by their frequencies so even when a $\sigma^{-}$polarized probe, e.g., couples to the $|3\rangle$ $\rightarrow|0\rangle$ transition, it would fall outside the trigger transparency window already for Zeeman splittings of few $\gamma$ 's yielding no cross-Kerr modulation.

${ }^{3}$ For typical Zeeman splittings of $0.13 \gamma / \mathrm{G}$, magnetic fields of the order of 150-250 G are here required. These field strengths are common in recent cold atoms experiments.

${ }^{4}$ Because symmetry $\left|5 P_{3 / 2}, F=2, m=0\right\rangle$, lying about $26 \gamma$ above the level $|0\rangle$, is the nearest resonant level to which a wrong probe could couple to, the two detuning cases which we examine in our predictions, namely $\delta_{1} \simeq \delta_{3} \simeq 10 \gamma$ and $\delta_{1} \simeq \delta_{3} \simeq 20 \gamma$, would require, respectively, Zeeman splittings of about $20 \gamma$ and $30 \gamma$.
} 

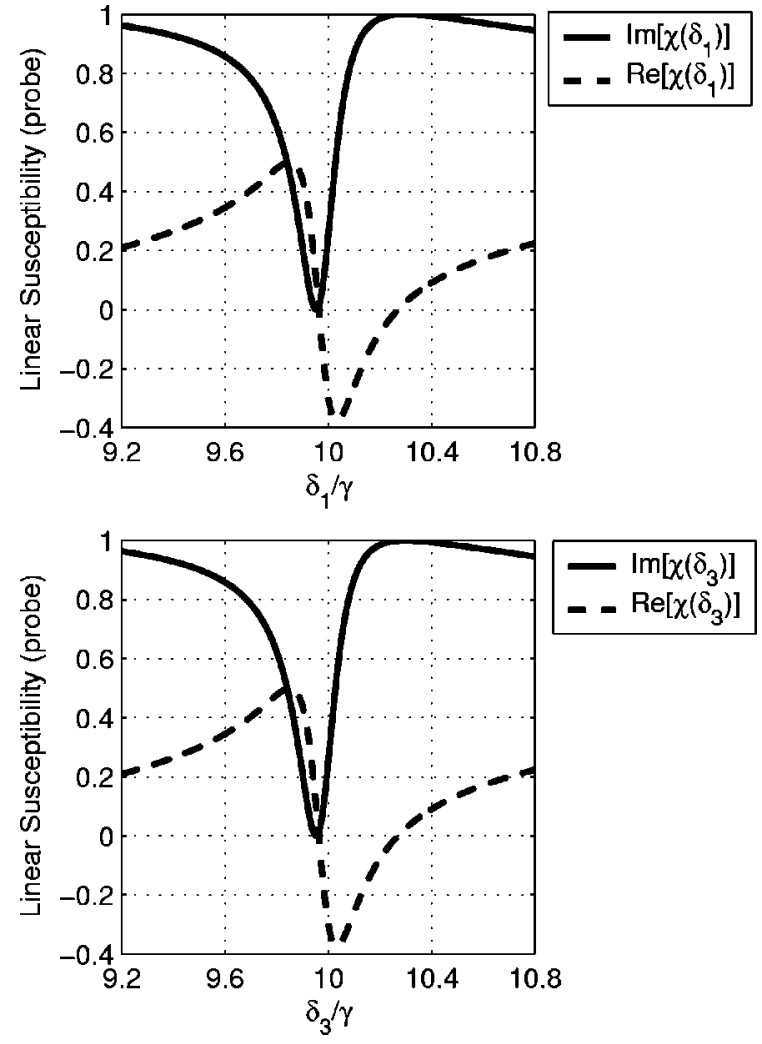

FIG. 3. Probe absorption and dispersion $\chi\left(\delta_{1}\right)$ $=\hbar \epsilon_{0} \chi_{P} /\left(\mathcal{N}\left|\boldsymbol{\mu}_{P}\right|^{2}\right)=\left(\rho_{10} / \Omega_{P}\right)_{s s}\left[\gamma^{-1}\right]$ (upper frame) vs the probe detuning $\delta_{1} / \gamma$ when $\delta_{3}=10.02 \gamma$ and $\delta_{2}=10 \gamma$. Trigger absorption and dispersion $\chi\left(\delta_{3}\right)=\hbar \epsilon_{0} \chi_{T} /\left(\mathcal{M}\left|\boldsymbol{\mu}_{T}\right|^{2}\right)\left(\rho_{30} / \Omega_{T}\right)_{s s}\left[\gamma^{-1}\right]$ (lower frame) vs the trigger detuning $\delta_{3} / \gamma$ when $\delta_{1}=10.01 \gamma$ and $\delta_{2}=10 \gamma$. In both cases we take the Rabi frequencies as $\Omega_{P}=\Omega_{T}=\gamma, \Omega=4.5 \gamma$.

can be coupled to and the fields acquire the trivial vacuum phase shift $\phi_{0}^{j}=k_{j} l, j=P, T$.

A probe and a trigger polarized single photon wave packets form a qubit [29]

$$
\left|\psi_{i}\right\rangle=\alpha_{i}^{+}\left|\sigma^{+}\right\rangle_{i}+\alpha_{i}^{-}\left|\sigma^{-}\right\rangle_{i}, \quad i=\{P, T\} .
$$

This qubit is a superposition of two circularly polarized states

$$
\left|\sigma^{ \pm}\right\rangle_{i}=\int d \omega \xi_{i}(\omega) a_{ \pm}^{\dagger}(\omega)|0\rangle
$$

where $\xi_{i}(\omega)$ is a Gaussian frequency distribution of incident wave packets, centered at frequency $\omega_{i}$. Traversing the atomic medium of length $l$, the photon field operator undergoes a transformation

$$
a_{ \pm}(\omega) \rightarrow a_{ \pm}(\omega) \exp \left\{i \frac{\omega}{c} \int_{0}^{l} d z n_{ \pm}(\omega, z)\right\} .
$$

The real part of the refractive index $n_{ \pm}$can be assumed to vary slowly over the bandwidth of the wave packets $n_{ \pm}(\omega, z) \approx n_{ \pm}\left(\omega_{i}, z\right)$, giving rise to a phase shift on a circularly polarized states $\left|\sigma^{+}\right\rangle_{i} \rightarrow e^{-i \phi_{ \pm}^{i}}\left|\sigma^{ \pm}\right\rangle_{i}$, where

$$
\phi_{ \pm}^{i}=\frac{\omega}{c} \int_{0}^{l} d z n_{ \pm}\left(\omega_{i}, z\right)
$$

For a Gaussian trigger pulse of time duration $\tau_{T}$ and Rabi frequency $\Omega_{T}$, moving with group velocity $v_{g}^{T}$, the nonlinear probe phase shift can be written as

$$
\phi_{\text {nlin }}^{P}=k_{P} l \frac{\pi^{3 / 2} \hbar^{2}\left|\Omega_{T}\right|^{2}}{4\left|\boldsymbol{\mu}_{T}\right|^{2}} \frac{\operatorname{erf}\left[\zeta_{\mathrm{P}}\right]}{\zeta_{P}} \operatorname{Re}\left[\chi_{P}^{(3)}\right],
$$

where $\zeta_{P}=\left(1-v_{g}^{P} / v_{g}^{T}\right) \sqrt{2} l / v_{g}^{P} \tau_{T}$. The trigger shift is obtained upon interchanging $P \leftrightarrow T$ in the equation above, namely

$$
\phi_{\text {nlin }}^{T}=k_{T} l \frac{\pi^{3 / 2} \hbar^{2}\left|\Omega_{P}\right|^{2}}{4\left|\boldsymbol{\mu}_{P}\right|^{2}} \frac{\operatorname{erf}\left[\zeta_{\mathrm{T}}\right]}{\zeta_{T}} \operatorname{Re}\left[\chi_{T}^{(3)}\right],
$$

where also the expression for $\zeta_{T}$ has to be changed accordingly.

The truth table for a polarization QPG that uses our tripod configuration reads as

$$
\begin{gathered}
\left|\sigma^{-}\right\rangle_{P}\left|\sigma^{-}\right\rangle_{T} \rightarrow e^{-i\left(\phi_{0}^{P}+\phi_{\text {lin }}^{T}\right)}\left|\sigma^{-}\right\rangle_{P}\left|\sigma^{-}\right\rangle_{T}, \\
\left|\sigma^{-}\right\rangle_{P}\left|\sigma^{+}\right\rangle_{T} \rightarrow e^{-i\left(\phi_{0}^{P}+\phi_{0}^{T}\right)}\left|\sigma^{-}\right\rangle_{P}\left|\sigma^{+}\right\rangle_{T}, \\
\left|\sigma^{+}\right\rangle_{P}\left|\sigma^{+}\right\rangle_{T} \rightarrow e^{-i\left(\phi_{\text {lin }}^{P}+\phi_{0}^{T}\right)}\left|\sigma^{+}\right\rangle_{P}\left|\sigma^{+}\right\rangle_{T}, \\
\left|\sigma^{+}\right\rangle_{P}\left|\sigma^{-}\right\rangle_{T} \rightarrow e^{-i\left(\phi_{+}^{P}+\phi_{-}^{T}\right)}\left|\sigma^{+}\right\rangle_{P}\left|\sigma^{-}\right\rangle_{T},
\end{gathered}
$$

with $\phi_{+}^{P}=\phi_{\text {lin }}^{P}+\phi_{\text {nlin }}^{P}$ and $\phi_{-}^{T}=\phi_{\text {lin }}^{T}+\phi_{\text {nlin }}^{T}$ and where the conditional phase shift $s$ given by

$$
\phi=\phi_{+}^{P}+\phi_{-}^{T}-\phi_{\text {lin }}^{P}-\phi_{\text {lin }}^{T} .
$$

Notice that only the nonlinear shifts contribute to $\phi$. The truth table of Eqs. (18) differs from that of Ottaviani et al. [29] only for the presence of a linear phase shift for the trigger, arising from the fact that also level $|3\rangle$ is populated with one-half of the atoms.

In the ${ }^{87} \mathrm{Rb}$ level configuration chosen above, the decay rates are equal $\gamma_{j 0}=\gamma$ and we take for simplicity equal and small dephasing rates $\gamma_{i j} \simeq \gamma_{d}=10^{-2} \gamma$. For $\Omega_{P} \approx \Omega_{T}=0.1 \gamma$, $\Omega=\gamma$, and detunings $\delta_{1}=20.01 \gamma, \delta_{2}=20 \gamma, \delta_{3}=20.02 \gamma$ we obtain a conditional phase shift of $\pi$ radians over an interaction length $l=1.6 \mathrm{~mm}$ at a density $\mathcal{N}=3 \times 10^{13} \mathrm{~cm}^{-3}$. With these parameters, group velocities are essentially the same, giving $\operatorname{erf}\left[\zeta_{\mathrm{P}}\right] / \zeta_{\mathrm{P}}=\operatorname{erf}\left[\zeta_{\mathrm{T}}\right] / \zeta_{\mathrm{T}} \approx 2 / \sqrt{\pi}$. This choice of parameters corresponds to the case where probe and trigger have a mean amplitude of about one photon when the beams are tightly focused $(\sim 1 \mu \mathrm{m})$ and with a time duration in the microsecond scale.

In addition, it is worth noting that a classical phase gate could be implemented by using more intense probe and trigger pulses. For Rabi frequencies $\Omega_{P} \approx \Omega_{T}=\gamma, \Omega=4.5 \gamma$, and detunings $\delta_{1}=10.01 \gamma, \delta_{2}=10 \gamma, \delta_{3}=10.02 \gamma$, a conditional phase shift of $\pi$ radians, over the interaction length $l$ $=0.7 \mathrm{~cm}$, density $\mathcal{N}=3 \times 10^{12} \mathrm{~cm}^{-3}$ is obtained. Again, with these parameters, group velocities are the same. Probe and trigger susceptibilities corresponding to these parameter values are shown in Fig. 3. 
Both sets of parameters could be realized with cold atoms in a temporal dark SPOT of a MOT. Alternatively, a gas cell of standard length between 2.5 and $10 \mathrm{~cm}$ can be considered, but the increase in length is then compensated with a lower density. In this case one has to take care to use all copropagating laser beams to cancel the first order Doppler effect [13]. This shows that a demonstration of a deterministic polarization QPG can be made using present technologies.

As discussed above, we had to move away from the exact double EIT-resonance condition in order to have nonvanishing nonlinearities. Yet, under such condition the linear susceptibilities are not small and actually their contribution is dominant. The nonlinear-linear phase shift ratios are in fact given by

$$
\frac{\phi_{P}^{\text {nlin }}}{\phi_{P}^{\text {lin }}}=\frac{\left|\Omega_{T}\right|^{2}}{4} \times \operatorname{Re}\left[\frac{1}{\Delta_{13}}\left(\frac{\Delta_{12}}{\Delta_{10} \Delta_{12}-|\Omega|^{2}}+\frac{\Delta_{23}}{\Delta_{30}^{*} \Delta_{23}-|\Omega|^{2}}\right)\right],
$$

$$
\frac{\phi_{T}^{\text {nlin }}}{\phi_{T}^{\text {lin }}}=\frac{\left|\Omega_{P}\right|^{2}}{4} \times \operatorname{Re}\left[\frac{1}{\Delta_{13}^{*}}\left(\frac{\Delta_{12}}{\Delta_{10}^{*} \Delta_{12}^{*}-|\Omega|^{2}}+\frac{\Delta_{23}^{*}}{\Delta_{30}^{*} \Delta_{23}^{*}-|\Omega|^{2}}\right)\right],
$$

which turn out to be of the order $\sim 1 / 43$ for the first (quantum) set of parameters and $\sim 1 / 64$ for the second (semiclassical) set of parameters. This means that under the optimal conditions corresponding to a $\pi$ conditional phase shift, the total phase shift in each input-output transformation is very large, of the order of $45 \pi$ and $65 \pi$, respectively.

The experimental demonstration of our QPG mechanism relies on the precision with which conditional phase shifts can be determined and hence it is important to work with small errors in the measurement of a phase difference. These errors mainly originate from fluctuations of the laser intensities and detunings. In particular, $1 \%$ intensity fluctuations yield an error of about $4 \%$ on the value of the phase. Fluctuations of the relative detuning can be instead overcome by taking all lasers tightly phase locked to each other. Other important sources of error include dephasing of the groundstate coherences, whose main effect is to induce absorption. Non-negligible absorption implies a nonzero gate failure probability (one or both qubits missing at the output) making therefore the present quantum gate, which is deterministic in principle, a probabilistic gate. Figure 4 shows that for typically small dephasings $\gamma_{d} / 2 \pi \sim 10 \mathrm{kHz}$, or $\gamma_{d} \sim 10^{-2} \gamma$, the degree of absoprtion remains still fairly low. It should be

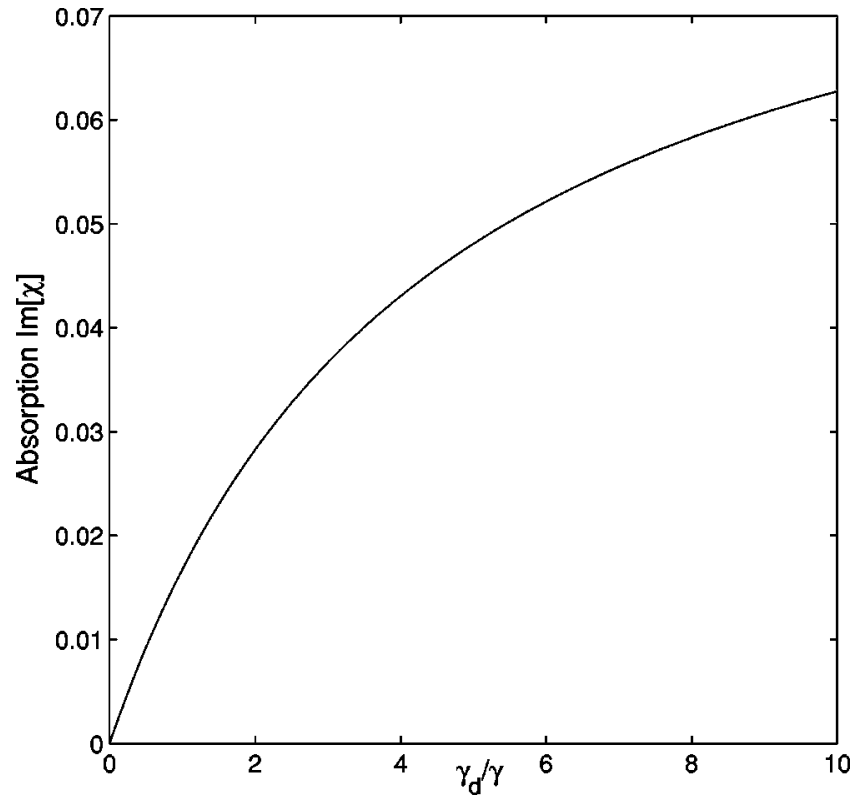

FIG. 4. Probe absorption (scaled) at the center of probe transparency window, plotted against the dephasing rate, for $\Omega_{P}=\Omega_{T}$ $=\gamma, \Omega=4.5 \gamma, \delta_{j}=0$.

mentioned that this holds for $\Omega \sim \gamma$, while for weaker control fields even smaller dephasings are required to keep absorption negligible.

\section{CONCLUSION}

In this paper we have studied the nonlinear response of a four-level atomic sample in a tripod configuration to an incident probe and trigger field. The resulting large cross-Kerr modulation between probe and trigger enables one to implement a phase gate with a conditional phase shift of the order of $\pi$. The main advantage of our proposal lies in its experimental feasibility which has been assessed through a detailed study of the requirements needed to observe such a large shift in a cold atomic sample of ${ }^{87} \mathrm{Rb}$ atoms.

\section{ACKNOWLEDGMENTS}

We acknowledge enlightening discussions with $\mathrm{P}$. Grangier, F. T. Arecchi, and M. Inguscio. We greatly acknowledge support from the MURST (Actione Integrada Italia-Spagna), the MIUR (PRIN 2001 Quantum Communications with Slow Light), and by MCyT and FEDER (Project No. BFM2002-04369-C04-02).
[1] M. A. Nielsen and I. L. Chuang, Quantum Computation and Quantum Information (Cambridge University Press, Cambridge, England, 2000).

[2] N. Gisin, G. Ribordy, W. Tittel, and H. Zbinden, Rev. Mod. Phys. 74, 145 (2002).

[3] F. Grosshans, G. V. Assche, J. Wenger, R. Brouri, N. J. Cerf, and P. Grangier, Nature (London) 421, 238 (2003).

[4] D. Bouwmeester, J.-W. Pan, K. Mattle, M. Eibl, H. Weinfurter, and A. Zeilinger, Nature (London) 390, 575 (1997).

[5] D. Boschi, S. Branca, F. DeMartini, L. Hardy, and S. Popescu, Phys. Rev. Lett. 80, 1121 (1998).

[6] A. Furusawa, J. L. Srensen, S. L. Braunstein, C. A. Fuchs, H. 
J. Kimble, and E. S. Polzik, Science 282, 706 (1998).

[7] W. P. Bowen, N. Treps, R. Schnabel, and P. K. Lam, Phys. Rev. Lett. 89, 253601 (2002).

[8] T. C. Zhang, K. W. Goh, C. W. Chou, P. Lodahl, and H. J. Kimble, Phys. Rev. A 67, 033802 (2003).

[9] E. Knill, R. Laflamme, and G. J. Milburn, Nature (London) 409, 46 (2001).

[10] J. L. O'Brien, G. J. Pryde, A. G. White, T. C. Ralph, and D. Branning, Nature (London) 426, 264 (2003).

[11] K. Sanaka, T. Jennewein, J.-W. Pan, K. Resch, and A. Zeilinger, Phys. Rev. Lett. 92, 017902 (2004).

[12] K. J. Boller, A. Imamoğlu, and S. E. Harris, Phys. Rev. Lett. 66, 2593 (1991).

[13] E. Arimondo, in Progress in Optics, edited by E. Wolf (Elsevier Science, Amsterdam, 1996), Vol. XXXV, p. 257.

[14] S. E. Harris, Phys. Today 50, 36 (1997).

[15] L. V. Hau, S. E. Harris, Z. Dutton, and C. H. Berhoozi, Nature (London) 397, 594 (1999).

[16] H. Schmidt and A. Imamoğlu, Opt. Lett. 21, 1936 (1996).

[17] H. Kang and Y. Zhu, Phys. Rev. Lett. 91, 093601 (2003).

[18] A. Imamoğlu, H. Schmidt, G. Woods, and M. Deutsch, Phys. Rev. Lett. 79, 1467 (1997).

[19] P. Grangier, D. F. Walls, and K. M. Gheri, Phys. Rev. Lett. 81, 2833 (1998).

[20] S. Rebić, S. M. Tan, A. S. Parkins, and D. F. Walls, J. Opt. B: Quantum Semiclassical Opt. 1, 490 (1999).

[21] K. M. Gheri, W. Alge, and P. Grangier, Phys. Rev. A 60, R2673 (1999).

[22] A. D. Greentree, J. A. Vaccaro, S. R. de Echaniz, A. V. Durant, and J. P. Marangos, J. Opt. B: Quantum Semiclassical Opt. 2, 252 (2000).

[23] J.-F. Roch, K. Vigneron, P. Grelu, A. Sinatra, J.-P. Poizat, and P. Grangier, Phys. Rev. Lett. 78, 634 (1997).

[24] H. Wang, D. Goorskey, and M. Xiao, Phys. Rev. Lett. 87, 073601 (2001).

[25] M. S. Zubairy, A. B. Matsko, and M. O. Scully, Phys. Rev. A
65, 043804 (2002).

[26] A. B. Matsko, I. Novikova, G. R. Welch, and M. S. Zubairy, Opt. Lett. 28, 96 (2002).

[27] A. B. Matsko, I. Novikova, M. S. Zubairy, and G. R. Welch, Phys. Rev. A 67, 043805 (2003).

[28] A. D. Greentree, D. Richards, J. A. Vaccaro, A. V. Durrant, S. R. de Echaniz, D. M. Segal, and J. P. Marangos, Phys. Rev. A 67, 023818 (2003).

[29] C. Ottaviani, D. Vitali, M. Artoni, F. Cataliotti, and P. Tombesi, Phys. Rev. Lett. 90, 197902 (2003).

[30] S. Lloyd, Phys. Rev. Lett. 75, 346 (1995).

[31] M. D. Lukin and A. Imamoğlu, Phys. Rev. Lett. 84, 1419 (2000)

[32] M. D. Lukin and A. Imamoğlu, Nature (London) 413, 273 (2001).

[33] D. Petrosyan and G. Kurizki, Phys. Rev. A 65, 033833 (2002).

[34] Q. A. Turchette, C. J. Hood, W. Lange, H. Mabuchi, and H. J. Kimble, Phys. Rev. Lett. 75, 4710 (1995).

[35] K. J. Resch, J. S. Lundeen, and A. M. Steinberg, Phys. Rev. Lett. 89, 037904 (2002).

[36] R. Unanyan, M. Fleischhauer, B. W. Shore, and K. Bergmann, Opt. Commun. 155, 144 (1998).

[37] E. Paspalakis and P. L. Knight, J. Opt. B: Quantum Semiclassical Opt. 4, S372 (2002).

[38] E. Paspalakis, N. J. Kylstra, and P. L. Knight, Phys. Rev. A 65, 053808 (2002).

[39] E. Paspalakis and P. L. Knight, J. Mod. Opt. 49, 87 (2002).

[40] Y. P. Malakyan, e-print quant-ph/0112058.

[41] D. Petrosyan and Y. P. Malakyan, e-print quant-ph/0402070.

[42] D. F. Walls and G. J. Milburn, Quantum Optics (Springer, Berlin, 1994).

[43] W. Ketterle, K. B. David, M. A. Joffe, A. Martin, and D. Pritchard, Phys. Rev. Lett. 70, 2253 (1993).

[44] M. Artoni, G. C. La Rocca, F. S. Cataliotti, and F. Bassani, Phys. Rev. A 63, 023805 (2001). 